\title{
Solar wind effects on Jupiter non-Io DAM emissions during Ulysses distant encounter (2003-2004)
}

\author{
E. Echer ${ }^{1}$, P. Zarka ${ }^{2}$, W. D. Gonzalez ${ }^{1}$, A. Morioka ${ }^{3}$, and L. Denis ${ }^{4}$ \\ 1 Instituto Nacional de Pesquisas Espaciais, Sao Jose dos Campos, SP, Brazil \\ e-mail: eecher@dge.inpe.br \\ 2 Observatoire de Paris, Meudon, France \\ 3 Planetary plasma and atmospheric research center, Tohoku University, Sendai, Japan \\ 4 Observatoire de Paris, Station de Radioastronomie, Nançay, France
}

Received 16 September 2009 / Accepted 11 May 2010

\begin{abstract}
Aims. We analyze solar wind data from the Ulysses spacecraft during the distant Jupiter encounter from 2003 November to 2004 March as well as Nançay decametric (DAM) radio observations non-controlled by Io.

Methods. The Ulysses solar wind data are balistically propagated towards Jupiter and are correlated with Nançay non-Io DAM emissions.

Results. It is found that the average solar wind dynamic pressure around the time of DAM emissions is 1.7 times higher than its average value during the Ulysses encounter. The occurrence of fast forward (FS) and reverse (RS) interplanetary shocks and heliospheric current sheet crossings (HCS) is correlated with the occurrence of non-Io DAM emissions. We note an enhanced probability of occurrence of non-Io DAM emissions after the expected arrival of FS, RS and HCS at Jupiter. However, about half emissions (54\%) did not seem to be associated with these interplanetary structures. We also note that the average duration and power of non-Io DAM emissions are enhanced during periods associated with those interplanetary structures.

Conclusions. From the results obtained in this work it seems that non-Io DAM emissions occur during intervals of enhanced solar wind dynamic pressure. Yet, there is no direct correlation between the non-Io DAM emissions duration or power versus the solar wind pressure values and the interplanetary shock Mach number.
\end{abstract}

Key words. planets and satellites: general - solar wind

\section{Introduction}

Jupiter's magnetosphere is the largest in the solar system. It differs from the Earth's magnetosphere mainly because of Jupiter's fast rotation and the influence of its moons. Furthermore, the solar wind dynamic pressure and interplanetary magnetic field (IMF) have lower values at the orbit of Jupiter than at that of Earth. Jupiter's magnetosphere has been studied by groundbased remote sensing since the 1950s, when its decameter (DAM) radio emissions were discovered (Burke \& Franklin 1955), and by several spacecraft flybys since the 1970s Pioneers10 and -11 , Voyagers- 1 and -2, Ulysses, Cassini, New Horizons and the Galileo orbiter (Bagenal et al. 2004).

Jupiter's magnetospheric activity can be (partly) monitored from the ground through radio-telescope observations. Jovian emissions, when observed from Earth, are dependent on the central meridian longitude (CML) of the observer and on the phase of Io (Carr et al. 1983). The moon Io exerts a strong electrodynamic influence on the Jovian magnetospheric activity, including its high latitude radio emission (Bigg 1964; Goldreich \& Lynden-Bell 1969; Crary \& Bagenal 1997; Gerard et al. 2006). As a consequence, part of this emission is controlled by the geometry of Io in relation to Jupiter and to the Earth, resulting in preferred pairs of CML-Io phase values for the occurrence of Io emissions (Carr et al. 1983; Boischot et al. 1987; Genova et al. 1989; Queinnec \& Zarka 1998).
There are also some emissions that are independent of Io (Bozyan \& Douglas 1976; Carr et al. 1983; Clarke et al. 2004). These type of emissions, called non-Io DAM, seem to respond to solar wind variations (Genova et al. 1989; Zarka \& Genova 1989). Together with their correlation with lower frequency radio components localized by Voyager-1, 2 and Ulysses (Genova et al. 1987; Ladreiter et al. 1994) and with UV aurora (Prangé et al. 1993), this suggests that non-Io DAM originates from Jovian magnetic field lines at a higher invariant latitude than the Io DAM emissions. However, the source distribution in longitude is unknown. It has been considered that non-Io DAM and HOM emissions could be the same radio component, but emitted at different latitudes, i.e., along field lines with magnetic-Lshell $=7$ to 9 for HOM, and L-shell $>9$ for non-Io DAM (Zarka 1998; Zarka et al. 2004).

The Jovian DAM radiation is emitted in the electromagnetic $\mathrm{X}$ mode at frequencies approximately equal to the local electron cyclotron frequency from northern and southern high magnetic latitude source regions (Zarka 1998). The main mechanism responsible for the emissions is thought to be the electron cyclotron maser instability; electrons moving at moderately relativistic velocities, (about $0.1 c$ ) are guided by the magnetic field of Jupiter towards high magnetic latitudes. There the emission occurs at or near the local cyclotron frequency of electrons at the expense of their perpendicular energy. The emission is beamed at wide angles in a hollow conical beam to the local magnetic 
field vector (e.g., Carr et al. 1983; Goldstein \& Goertz 1983; Zarka 1998). The radiation is highly polarized, $100 \%$ elliptical above $10 \mathrm{MHz}$ (Zarka 1998). The observed elliptical polarization sense is generally right-hand ( $\mathrm{RH})$ from the northern hemisphere and left-hand (LH) from the southern hemisphere. Righthand emission is dominant, as observed from the Earth (e.g., Leblanc et al. 1993), possibly due to the stronger magnetic field at Jovian northern latitudes (Acuna et al. 1983). Ground-based observations of the DAM emissions are limited toward the lower frequencies at about $10 \mathrm{MHz}$ by the effects of Earth's ionosphere. The upper limit of the DAM spectral range is the natural limit of the emissions, around $40 \mathrm{MHz}$. This limit is the electron gyrofrequency at the altitude at which precipitating electrons become lost to the ionosphere (Genova et al. 1989; Zarka 1998).

At first sight, it could be expected that compression of the magnetosphere by enhanced solar wind pressure causes an auroral intensification. However, recent theoretical models (Cowley \& Bunce 2001; Southwood \& Kivelson 2001) predicted the opposite, namely an anticorrelation between solar wind pressure and auroral emissions. The brightness of the main auroral oval should be anti-correlated with the solar wind dynamic pressure. During magnetospheric compression (expansion), the angular velocity of the equatorial plasma increases (decreases) because of conservation of the angular momentum, and the corresponding magnetosphere-ionosphere coupling currents will then be reduced (enhanced), leading to a decrease (increase) in auroral emission intensity.

However, the association between solar wind pressure and auroral intensity is more complex than these simple models suggest. Cowley et al. (2007) constructed a model of the plasma flow, magnetosphere-ionosphere coupling currents, and auroral precipitation in Jupiter's magnetosphere, examining how they respond to compressions and expansions of the magnetosphere driven by changes in solar wind dynamic pressure. Their results show that the magnitude of the field-aligned current is also dependent on the timescale of the response of the ionospheric neutrals relative to the ions. Cowley et al. (2007) found that for a few hours following an impulsive compression, the main oval will dim because of the increased angular velocity of the equatorial plasma, and the emission at the open-closed field line boundary will brighten because of the increased flow shear across the boundary. As discussed by Nichols et al. (2009), if the compression is strong enough to induce super-rotation, a condition that is achieved by more modest compressions if the neutral atmosphere is unresponsive, then the current system will reverse and instead produce emission on field lines mapping to the outer magnetosphere between the middle magnetosphere and the magnetopause. After a few hours the system will revert to the steady state and the usual sense of the current system will be restored, with smaller currents and thus dimmer main oval auroras.

From the observational point of view, evidence has been presented that shows a significant effect of an impinging solar wind compression region or solar wind enhanced pressure on Jupiter's auroral activity. Solar wind effects on Jupiter auroral radio (Barrow 1978, 1979; Terasawa et al. 1978; Barrow et al. 1986; Genova et al. 1987; Zarka \& Genova 1989; Prangé et al. 1993; Morioka et al. 2002; MacDowall et al. 2006) and UV emissions have been widely studied (Clarke et al. 2004, 2009; Gurnett et al. 2002; Prangé et al. 1993; Prangé et al. 2004; Nichols et al. 2007, 2009). Terasawa et al. (1978) showed an association between solar wind high pressure events, using propagated solar wind data from $1 \mathrm{AU}$ to $5 \mathrm{AU}$, employing a MHD model, and non-Io DAM radio emissions. Gurnett et al. (2002) showed enhanced auroral UV and radio (hectometer) power during the passage of three shocks by Jupiter. Prangé et al. (2004) studied a single solar wind event (shock) that was traced from $1 \mathrm{AU}$ and possibly corresponded to auroral brightening at Jupiter and Saturn. Nichols et al. (2007) showed that a solar wind compression region observed by Cassini in its approach to Jupiter induced a significant brightening of the UV auroras. However, although compression regions are characterized by higher overall dynamic pressure, they also contain many transient expansions and contractions. Therefore, and because of the uncertainty in the propagation of solar wind, it was likewise unclear whether the brightening was in response to an expansion or contraction.

Similar brightening of the main auroral oval was observed by Nichols et al. (2009) although they had to rely on an MHD propagation of the solar wind from Earth. Clarke et al. (2009) studied Jupiter's auroras with the Hubble Space Telescope in combination with a MHD-propagation model of the solar wind from Earth's orbit, and determined the auroral response to the arrival of interplanetary fast forward (FS) and reverse (RS) shocks. They noted that the auroral power generally increased in response to forward shocks, albeit with uncertainty in the arrival times, while RS showed no associated auroral brightening. However, the correlation between auroral radio emission and solar wind pressure is weaker for Jupiter than for Saturn and Earth, probably because the fact that the Jovian magnetosphere is controlled mainly by its internal dynamics.

During the end of 2003 and beginning of 2004, the spacecraft Ulysses underwent the so called distant encounter with Jupiter. This distant encounter is an opportunity to use solar wind data relatively close to Jupiter to study the magnetospheric response, namely auroral radio emissions, to solar wind variations. At that time, an intensification of radio emissions in the bKOM, nKOM and non-thermal continuum have been found with Ulysses URAP instrument (MacDowall et al. 2006). The Nançay radio-telescope was operating normally during that period and a catalog of non-Io DAM radio emissions has been derived by one of us (A. Morioka). Thus the objective of the present paper is to study the solar wind conditions, using Ulysses data propagated to Jupiter, around the time of the Nançay nonIo DAM radio storms, to characterize the interplanetary medium and to identify possible causes of those radio emissions.

\section{Nançay decametric array and Ulysses solar wind data}

From 2003 October to 2004 March, Ulysses spacecraft moved from 0.8-1.2 AU distance from Jupiter, from high Jovian northern latitudes $\left(<75^{\circ}\right)$. The second encounter with Jupiter occurred with a closest approach in 04 February 2004, at a range of $0.8 \mathrm{AU}$, or $1683 R \mathrm{j}$, and a Jovian latitude of $49^{\circ}$ (MacDowall et al. 2006; Anagnostopoulos et al. 2009, and Ulysses distant encounter homepage, ulysses.jpl .nasa.gov/science/jupitertwo.html).

The Nançay decameter array (NDA) has operated since 1977, routinely observing Jupiter's decametric radio emissions (Boischot et al. 1980). The NDA is a phased array consisting of 144 helix conical log-periodic antennas: 72 sensitive to RH circular polarization and 72 to $\mathrm{LH}$ polarization. The frequency range that can be observed is $10-100 \mathrm{MHz}$. Each of the two arrays has a total collecting area of about $3500 \mathrm{~m}^{2}$ at frequencies below $25 \mathrm{MHz}$, allowing for the detection in $1 \mathrm{~s}$ of a 1000 Jansky source in a $50 \mathrm{kHz}$ bandwidth. The gain is $25 \mathrm{~dB}$ above $25 \mathrm{MHz}$. However, the main beam has a poor spatial resolution, $6^{\circ} \times 10^{\circ}$. 
This does not permit the resolution of the source of Jupiter's non-Io DAM emissions. This drawback is compensated though by the broad-band, high frequency and time resolutions of the NDA observations. The telescope can observe Jupiter from four hours before until four hours after its meridian transit. The frequency covered for Jupiter observations is $10-40 \mathrm{MHz}$. The data are represented in dynamic spectra, i.e., intensity as a function of time and frequency.

Ulysses one-hour magnetic field and plasma data were used to study the solar wind at Jupiter's orbit. The Ulysses magnetic field instrument was described in Balogh et al. (1993) and the plasma instrument in Bame et al. (1993). The Ulysses list of solar wind structures, available from http://ulysses.jpl. nasa.gov/science/jupitertwo.html, was used to help identify the solar wind structures of interest. Higher resolution data were also used to help to identify the interplanetary FS and RS.

Ulysses solar wind plasma and magnetic field data weere ballistically propagated to Jupiter, taking into accout the radial and longitudinal separation between Ulysses and Jupiter.

$t=t_{0}+\frac{\Delta r}{V_{\mathrm{sw}}}+\frac{\Delta \phi}{\Omega_{\mathrm{s}}}$.

In Eq. (1), $t$ is the time of propagated solar wind at Jupiter, $t_{0}$ the time at Ulysses, $V_{\mathrm{sw}}$ is the solar wind speed, $\Omega_{\mathrm{s}}$ is the angular speed of Sun's rotation, $\Delta r$ the radial heliographic distance between Ulysses and Jupiter (0.8-1.2 AU), and $\Delta \phi$ their longitudinal separation $\left(\leq 10^{\circ}\right)$. Ballistic propagation under these conditions leads to an uncertainty on the arrival time at Jupiter less than one day (Prangé et al. 1993).

Between 2003 November and 2004 March, 37 non-Io DAM emissions were identified. We used the time of their occurrence to study the solar wind conditions at Jupiter. The duration and average power were also analyzed. The Nançay dynamic spectrograms for these 37 emissions were processed to remove calibration and interference effects (Prangé et al. 1993; Zarka et al. 2004). Then we calculated the average power over the frequency bandwidth of the emissions. One drawback of the Nançay data is that due to artificial interference sources, the average power of emissions could be calculated usually only above $15 \mathrm{MHz}$.

\section{Results}

Figure 1 shows the Ulysses solar wind speed and the distance from Ulysses to Jupiter in AU, and the heliocentric distance and latitude coordinates of the Ulysses spacecraft. Ulysses approached Jupiter from high Jovigraphic latitudes and Jupiter local times from about $12 \mathrm{~h}$ to $18 \mathrm{~h}$. The distance from Jupiter was from about 0.8 to $1.2 \mathrm{AU}$ (range). It can be seen from Fig. 1 that there are many potential shocks. At 5 AU, most shocks are associated with corotating interaction regions (CIRs) (e.g., Smith \& Wolfe 1976; Echer et al. 2010, and references therein), but the interplanetary remnants of coronal mass ejections (ICMEs) are also present, such as the big peak of solar wind speed reaching $1000 \mathrm{~km} \mathrm{~s}^{-1}$ in this data set (de Koning et al. 2005).

Figure 2 shows the distribution of the occurrence of non-Io DAM emissions in the CML Io phase plane. It can be seen that most emissions occur within CML $180^{\circ}-360^{\circ}$ (non-Io A and C). In Table 1 the date, starting times, duration, and average power of the non-Io DAM emissions are shown. Also listed in this table are solar wind parameters within \pm 1 day of the emission: solar wind dynamic pressure, and the type of structure: FS, RS and heliospheric current sheet crossings (HCS). Figure 3 shows

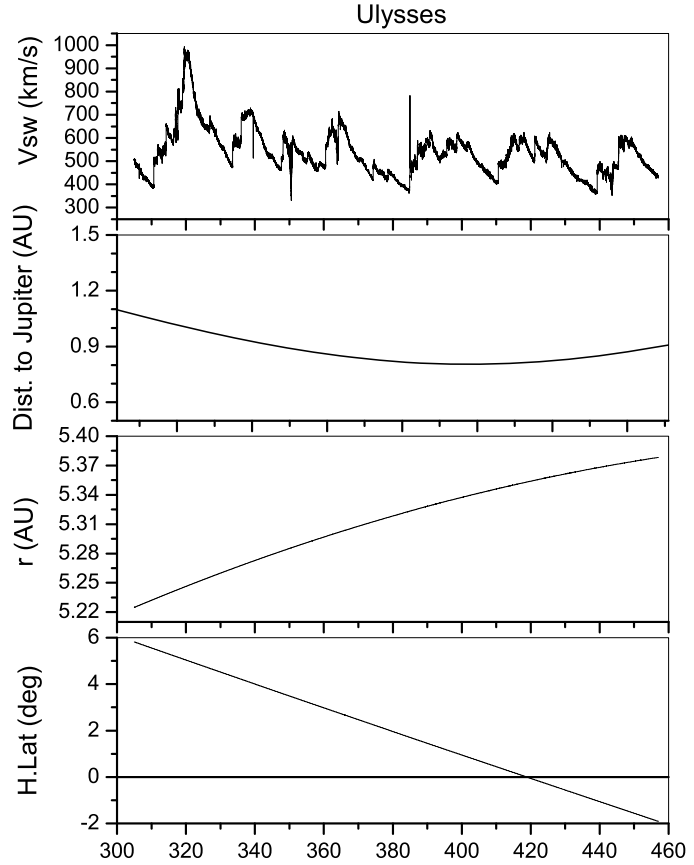

Fig. 1. Ulysses solar wind speed and heliographic coordinates: distance to Jupiter (AU), heliocentric distance (AU) and heliographic latitude ${ }^{(\circ)}$.

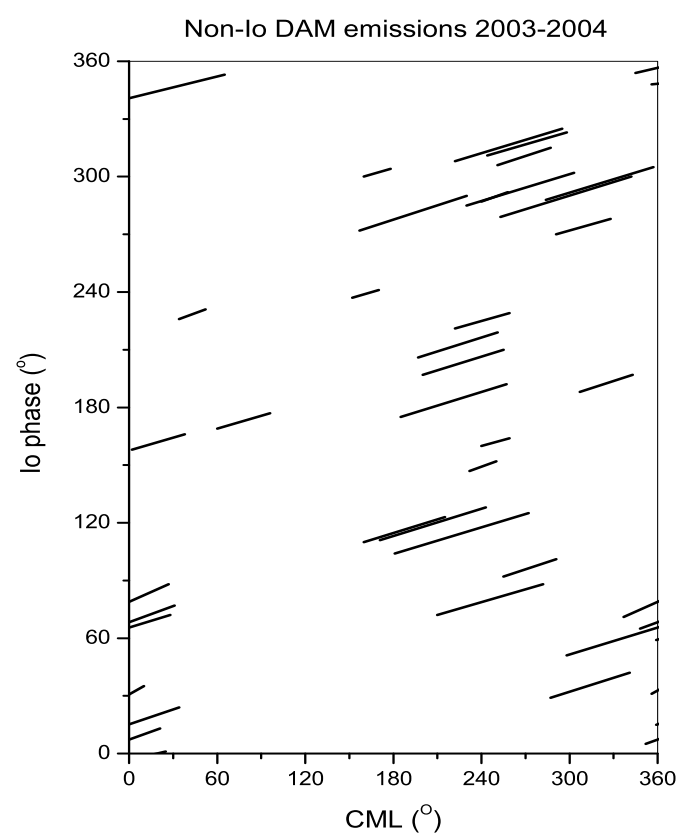

Fig. 2. Diagram of central meridian longitude (CML) versus Io phase for Nançay non-Io DAM emissions.

a histogram of the occurrence distribution of the duration and average power for the non-Io DAM emissions. Their duration is $74 \mathrm{~min}$ on average (median $=69 \mathrm{~min}$ ). The average power is $1.9 \times 10^{-15} \mathrm{~W} \mathrm{~m}^{-2}\left(\right.$ median $\left.=1.4 \times 10^{-15} \mathrm{~W} \mathrm{~m}^{-2}\right)$.

Figure 4 shows Ulysses solar wind data propagated to Jupiter according to Eq. (1). Only the dynamic pressure is shown here to correlate with Nançay emissions. The dashed lines show the occurrence of non-Io DAM emissions (their starting times are plotted). 
A\&A 519, A84 (2010)

Table 1. Nançay non-Io DAM emissions during the period 2003 November to 2004 March.

\begin{tabular}{|c|c|c|c|c|}
\hline Date/time & $\mathrm{d} T(\min )$ & Power $\left(10^{-15} \mathrm{~W} \mathrm{~m}^{-2}\right)$ & Psw (nPa) & IP structure \\
\hline 01/11/2003-07:04 & 17 & 1.40 & 0.06 & - \\
\hline 04/11/2003-04:18 & 34 & 1.47 & 0.02 & - \\
\hline 09/11/2003-03:53 & 67 & 0.57 & 0.40 & FS/HCS \\
\hline $11 / 11 / 2003-04: 52$ & 29 & 2.56 & 0.25 & $-\mathrm{RS}$ \\
\hline $14 / 11 / 2003-03: 54$ & 91 & 3.13 & 0.89 & $\mathrm{FS} / \mathrm{HCS} / \mathrm{HCS}$ \\
\hline $1611 / 2003-03: 45$ & 41 & 0.42 & 0.23 & FS/HCS \\
\hline $25 / 11 / 2003-04: 26$ & 84 & 0.52 & 0.04 & - \\
\hline 03/12/2003-03:10 & 101 & 5.35 & 0.14 & RS \\
\hline 15/12/2003-02:13 & 92 & 0.82 & 0.49 & FS/FS/HCS/HCS \\
\hline 24/12/2003-02:46 & 24 & 1.39 & 0.02 & - \\
\hline 27/12/2003-00:50 & 132 & 1.73 & 0.33 & FS/HCS \\
\hline 29/12/2003-03:30 & 90 & 1.48 & 0.23 & - \\
\hline 03/01/2004-01:55 & 151 & 0.19 & 0.02 & - \\
\hline 11/01/2004-00:30 & 146 & 0.78 & 0.28 & $\mathrm{HCS} / \mathrm{HCS}$ \\
\hline 15/01/2004-05:05 & 154 & 1.59 & 0.01 & - \\
\hline 19/01/2004-03:50 & 12 & 1.06 & 0.01 & - \\
\hline 23/01/2004-23:11 & 25 & 0.04 & 0.17 & RS \\
\hline 25/01/2004-03:12 & 48 & 1.07 & 0.11 & $\mathrm{RS} / \mathrm{RS} / \mathrm{FS}$ \\
\hline 27/01/2004-01:00 & 41 & 8.16 & 0.15 & FS \\
\hline 28/01/2004-04:11 & 18 & 3.27 & 0.16 & - \\
\hline 03/02/2004-04:02 & 84 & 1.08 & 0.11 & FS/RS \\
\hline 07/02/2004-00:14 & 78 & 2.25 & 0.09 & FS/HCS \\
\hline 09/02/2004-01:38 & 128 & 1.48 & 0.05 & - \\
\hline $11 / 02 / 2004-22: 41$ & 69 & 1.50 & 0.03 & - \\
\hline 14/02/2004-01:00 & 58 & 0.10 & 0.03 & - \\
\hline $16 / 02 / 2004-02: 30$ & 101 & 2.75 & 0.37 & FS/HCS \\
\hline $17 / 02 / 2004-02: 55$ & 119 & 1.13 & 0.36 & HCS \\
\hline $21 / 02 / 2004-01: 46$ & 62 & 1.10 & 0.05 & - \\
\hline 23/02/2004-03:26 & 83 & 2.70 & 0.06 & - \\
\hline 28/02/2004-02:43 & 95 & 1.51 & 0.20 & HCS \\
\hline $28 / 02 / 2004-22: 45$ & 67 & 11.3 & 0.12 & - \\
\hline 02/03/2004-01:00 & 20 & 0.71 & 0.05 & - \\
\hline 05/03/2004-23:20 & 132 & 2.20 & 0.01 & - \\
\hline 13/03/2004-01:00 & 50 & 1.07 & 0.04 & - \\
\hline 16/03/2004-00:08 & 52 & 0.93 & 0.31 & $\mathrm{FS} / \mathrm{HCS}$ \\
\hline $24 / 03 / 2004-23: 52$ & 60 & 1.13 & 0.04 & - \\
\hline $31 / 03 / 2004-23: 58$ & 84 & 2.10 & 0.02 & - \\
\hline
\end{tabular}

Notes. Columns are emission date and starting time, duration, average solar wind dynamic pressure within \pm 1 day of the emission, and solar wind structures present within \pm 1 day of the emission: FS, fast forward shocks; RS, fast reverse shocks; HCS, heliospheric current sheet crossings.

Figure 5 shows Ulysses solar wind data propagated to Jupiter and Nançay radio emission starting times for the period 13-15 November 2003. At the time of the emission, high speed, density and IMF magnitude (not shown) are noted at Jupiter. This corresponds to the higher dynamic pressure interval observed close to a DAM emission seen in Fig. 5.

Figure 6 shows an example of a Nançay radio spectrogram, corresponding to the emission marked with dotted lines in Fig. 5. This was an RH emission starting at 0430 UT, 14 November 2003. Intense arc emissions are seen in the Nançay data up to $24 \mathrm{MHz}$.

Figure 7 shows a scatter plot between Nançay non-Io DAM emissions, duration, and average power versus solar wind dynamic pressure (top and bottom panels, respectively). It can be seen that there is a large scatter in these plots. Although there is some indication of longer durations for higher values of solar wind pressure, both correlations are not significant at a 95\% confidence level (t-test), with coefficients of $r=0.17$ and $r=0.05$. This shows almost no linear relation between the duration and intensity of emissions versus average solar wind pressure within \pm 1 day of the emission.
Figure 8 shows a superposed epoch analysis of Nançay nonIo DAM emissions and solar wind interplanetary structures: heliospheric current sheet crossings (top panel), fast reverse shocks (intermediate panel) and fast forward shocks (bottom panel). The 0 day corresponds to the time when the interplanetary structure is expected to have impinged on Jupiter's magnetosphere. The DAM emissions were summed up per 0.5 day bin, from 2.5 days before to 2.5 days after the interplanetary structure was expected to arrive at Jupiter. It can be seen that for FS and HCS, there is an increased occurrence of non-Io DAM emissions after the expected arrival of the interplanetary structure at Jupiter, while there is a more uniform distribution for RS.

Table 2 shows the average (median) dynamic pressure for the whole period and within \pm 1 day of the non-Io DAM emissions. It can be seen that emissions occurred during periods of highly enhanced solar wind dynamic pressure (about 1.7 times the whole interval average).

Table 3 shows the association of emissions with interplanetary structures (as listed in Table 1). Most of the emissions (20) are not associated with HCS, FS or RS within \pm 1 day. The most common structure is a combination of FS + HCS. Summing up 

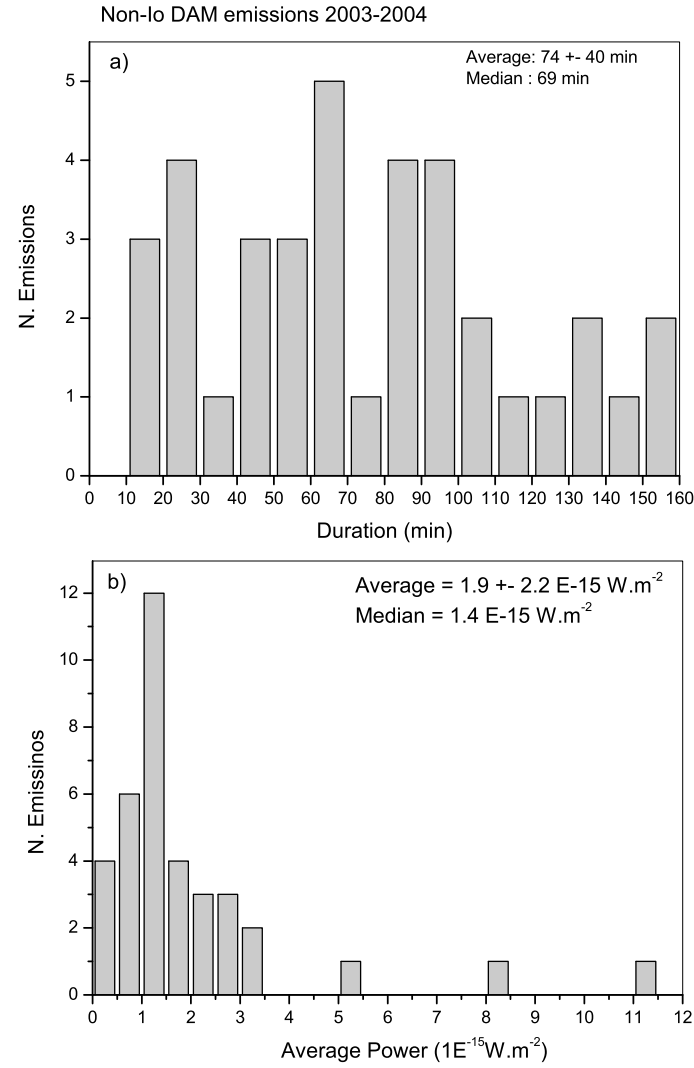

Fig. 3. Histogram of the distribution of the a) duration, and $\mathbf{b}$ ), average power, of Nançay non-Io DAM emissions.

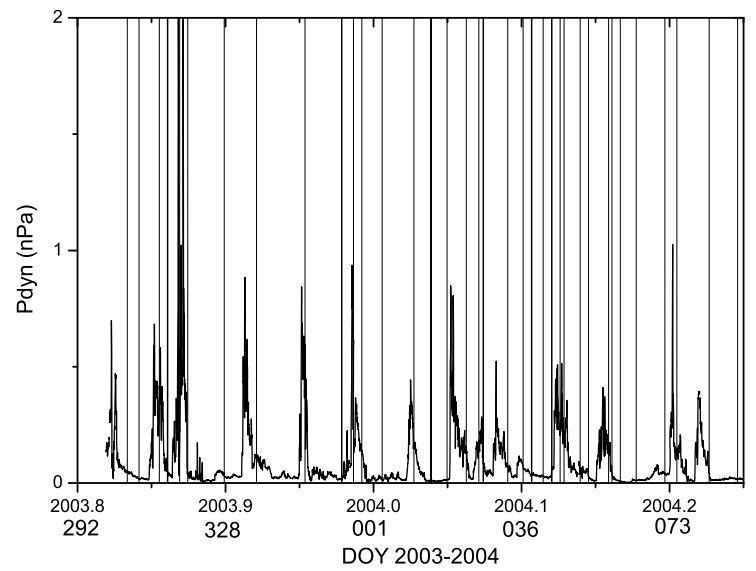

Fig. 4. Ulysses solar wind dynamic pressure propagated to Jupiter and emission starting times (dotted lines). The whole period of 2003 November to 2004 March is shown.

all events, there were 11 emissions associated with FS, 5 with RS and 11 with HCS. Considering only the presence of a single shock (FS or RS) within \pm 1 day of the emission, there were eight FS associated emissions and three RS associated emissions.

Table 4 shows the average duration for emissions associated with one interplanetary shock, multiple interplanetary shocks, HCS and unrelated to any interplanetary structure. It can be seen that the average solar wind dynamic pressure is enhanced for both FS and RS associated emissions. The FS associated emissions have a slightly longer duration and a higher avaerage power than the unrelated emissions. RS associated emissions have a

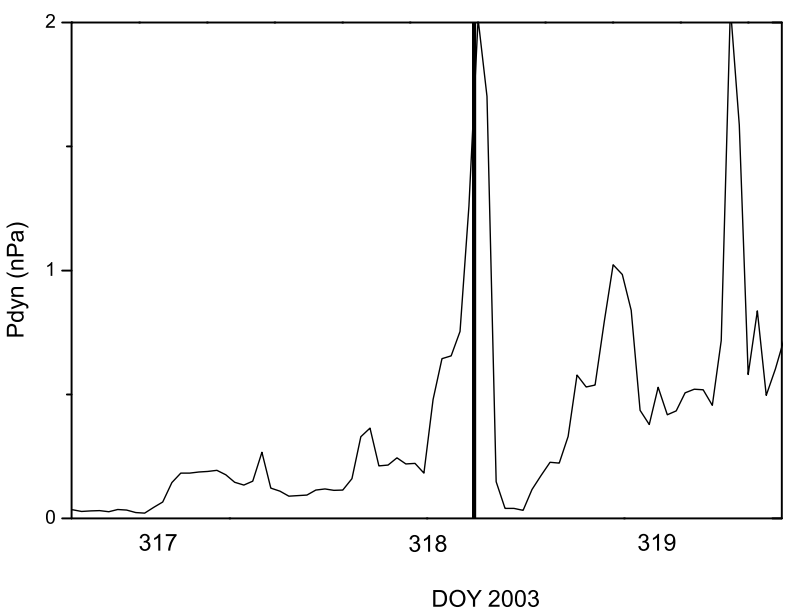

Fig. 5. Ulysses solar wind dynamic pressure propagated to Jupiter and Nançay radio emission starting times. The period 13-15 November 2003 is shown. At the time of the emission, high values of dynamic pressure and IMF magnitude are noted at Jupiter.

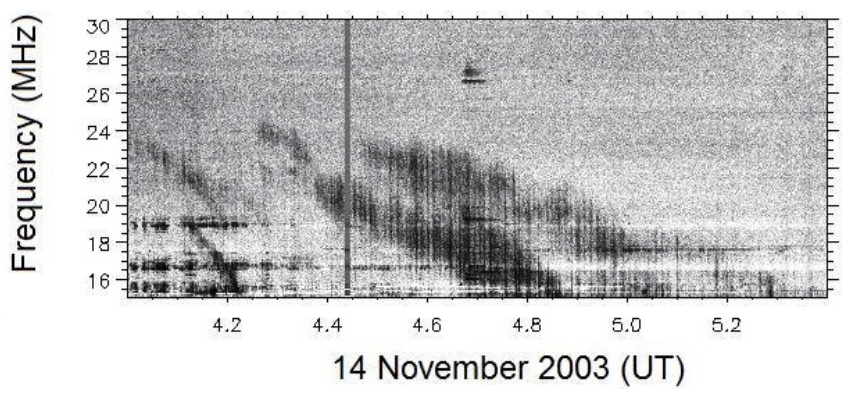

Fig. 6. Example of a Nançay radio spectrogram, corresponding to the second emission marked with dotted lines in Fig. 5. This was an RH emission starting at 0430 UT, 14 November 2003.
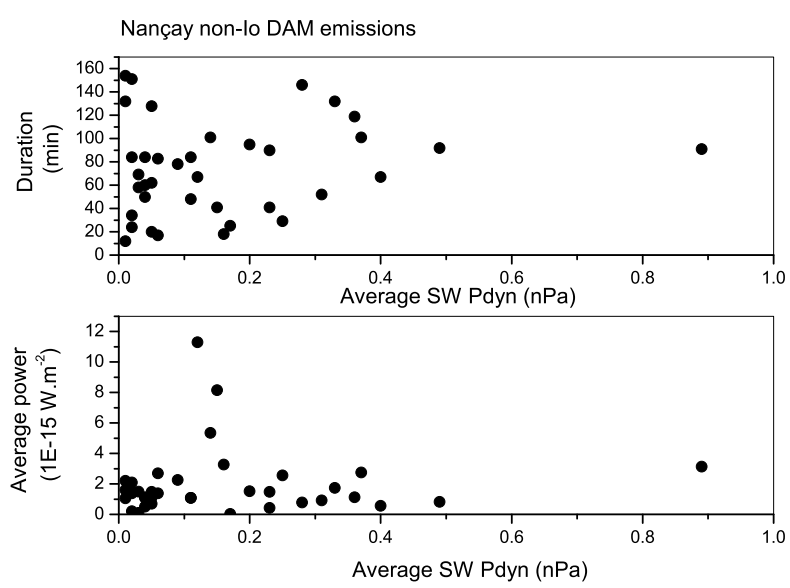

Fig. 7. Scatter plot between Nançay non-Io DAM emission duration and average power versus solar wind dynamic pressure.

shorter duration than both FS and unrelated emissions, but similar average power.

Shock analysis using the Rankine-Hugoniot conditions was applied to the interplanetary shocks (see Echer et al. 2003, 2010). Figure 9 shows the magnetosonic Mach number distribution calculated for the FS and RS shocks. It can be seen that 


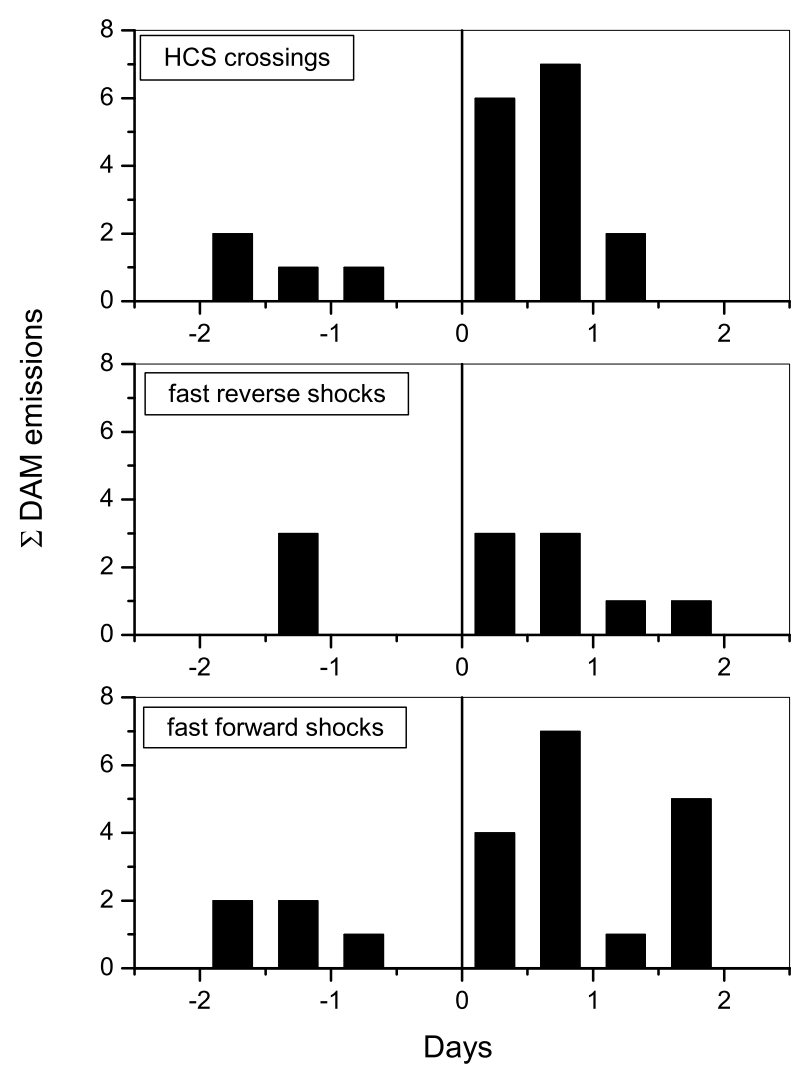

Fig. 8. Superposed epoch analysis of Nançay non-Io DAM emissions and solar wind interplanetary structures: heliospheric current sheet crossings (top panel), fast reverse shocks (intermediate panel) and fast forward shocks (bottom panel). The 0 day corresponds to the time when the interplanetary structure is detected at Jupiter. The DAM emissions were summed up per 0.5 day, from 2.5 days before to 2.5 days after the interplanetary structure be detected at Jupiter.

Table 2. Solar wind dynamic pressure for the whole interval (2003 November -2004 March) and within \pm 1 day of the starting time of Nançay DAM emissions.

\begin{tabular}{cccc}
\hline \hline Average(median) & Alll & $\pm 1 \mathrm{~d}$ & Ratio \\
\hline Pdyn & $0.09(0.04) \mathrm{nPa}$ & $0.15(0.07) \mathrm{nPa}$ & $1.67(1.75)$ \\
\hline
\end{tabular}

Notes. Also shown is the ratio of solar wind pressure for the intervals around the emission to the whole interval.

a small fraction of these FS are not shocks, but waves. On average, FS are slightly stronger (Mach number $=2.6$ ) than RS (Mach number $=2.4$ ).

Figure 10 shows the correlation between the duration (top panel) and power (bottom panel) versus the FS magnetosonic Mach number. The events selected correspond to emissions associated with a single FS, as explained before. The correlation coefficients ( 0.4 for duration and 0.08 for power) are statistically not significant at a $95 \%$ confidence level (t-test). This again indicates almost no linear relation between shock Mach number and emission average power and duration.

\section{Summary}

We studied non-Io DAM radio emissions observed by the Nançay decametric array during the Ulysses distant Jupiter
Table 3. Number of non-Io DAM emissions related to HCS, FS or RS.

\begin{tabular}{cc}
\hline \hline IP structure & N. emissions \\
\hline None & 20 \\
HCS & 2 \\
HCS+HCS & 1 \\
FS & 1 \\
FS+HCS & 6 \\
FS+HCS+HCS & 1 \\
FS+FS+HCS+HCS & 1 \\
FS+RS & 1 \\
FS+RS+RS & 1 \\
RS & 3 \\
\hline
\end{tabular}

Table 4. Average (medians) of radio emission duration, power, and solar wind pressure of Nançay non-Io DAM emissions for periods with FS, RS, HCS or no shocks within \pm 1 day of the emission.

\begin{tabular}{ccccc}
\hline \hline IP structure & $N$ & $\mathrm{~d} T(\min )$ & $P\left(10^{-15} \mathrm{~W} \mathrm{~m}^{-2}\right)$ & $P_{\mathrm{sw}}(\mathrm{nPa})$ \\
\hline FS & 8 & $75(67)$ & $2.5(1.7)$ & $0.27(0.23)$ \\
RS & 3 & $52(27)$ & $2.6(1.3)$ & $0.12(0.10)$ \\
HCS & 3 & $120(107)$ & $1.1(0.9)$ & $0.27(0.23)$ \\
Multiple S & 3 & $75(66)$ & $0.9(0.9)$ & $0.22(0.12)$ \\
None & 20 & $70(62)$ & $1.9(1.4)$ & $0.06(0.03)$ \\
\hline
\end{tabular}
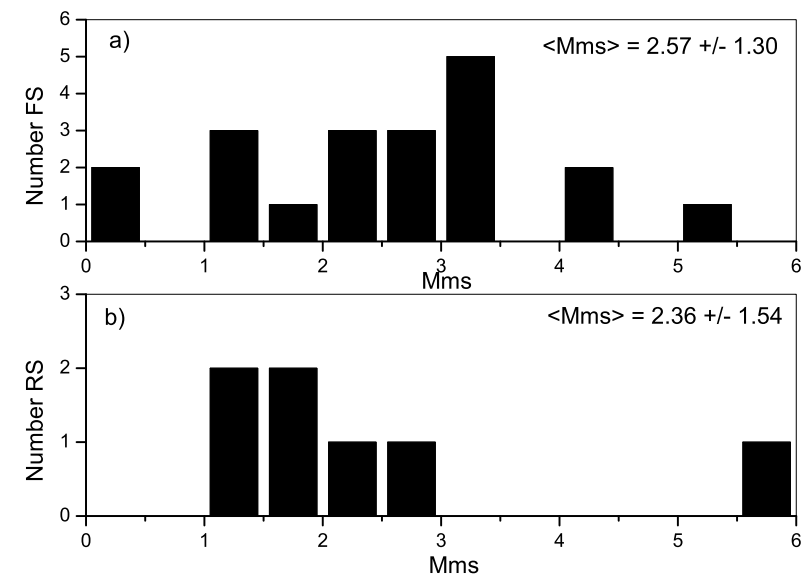

Fig. 9. Histogram of the distribution of shock magnetosonic Mach number for a) FS and b) RS.

encounter from 2003 November to 2004 March. The solar wind effects on the occurrence, duration, and power of non-Io DAM emissions were searched. We found that

1. Non-Io DAM emissions during the Ulysses distant encounter had an average (median) duration of 74(69) min. Their average power was $1.9(1.4) \times 10^{-15} \mathrm{~W} \mathrm{~m}^{-2}$.

2. Within \pm 1 day of emission occurrences, the solar wind dynamic pressure is enhanced by a factor of about 1.7 compared to the whole Ulysses encounter period.

3. There is no significant direct (linear) correlation between emission duration or average power and solar wind dynamic pressure around \pm 1 day of the emissions.

4. There is an enhanced probability of occurrence of non-Io DAM emissions for a period $<1$ day after interplanetary structures (HCS, FS and RS) are expected to arrive at Jupiter. 

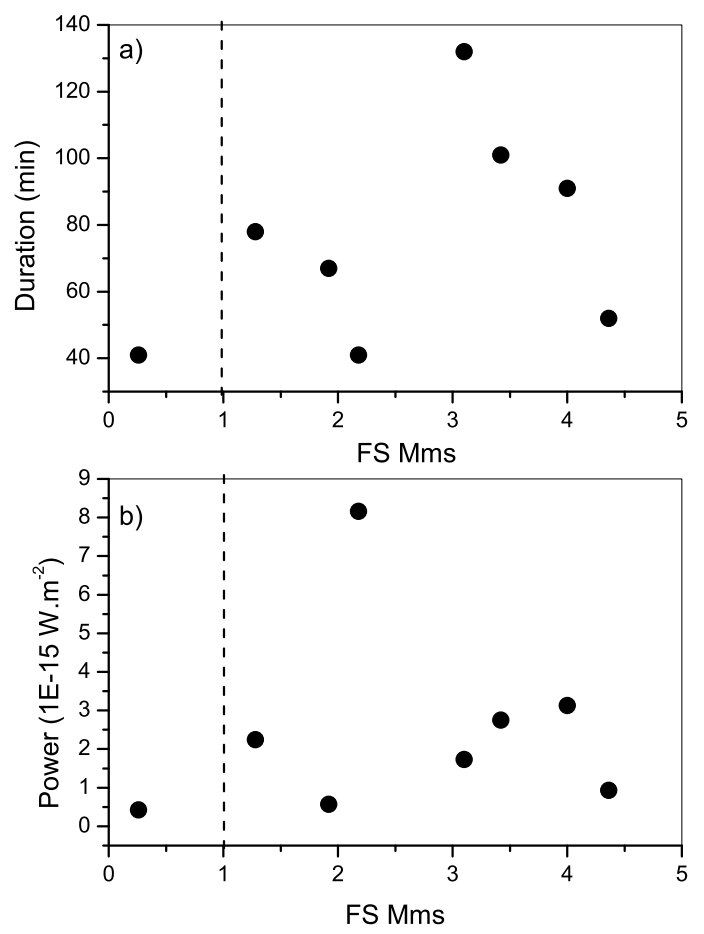

Fig. 10. Scatter plot between Nançay non-Io DAM emission duration and average power versus FS magnetosonic Mach number. The dashed line $(M=1)$ shows the limit of fast forward shock and wave cases.

5. About half of the emissions (54\%) do not have an associated interplanetary structure. The most usual structure was the combination of FS and HCS (22\%).

6. For emissions associated with FS (8) and RS (3), the solar wind dynamic pressure is much enhanced (4.5 and 2 times higher, respectively) as compared to the emissions unrelated with interplanetary structures. Emissions related to FS show higher power and longer durations (1.3 higher and 1.1 times longer) than the emissions unrelated to interplanetary structures), while RS related emissions have higher power but shorter duration.

7. There is no significant correlation between DAM emission duration and average power and shock magnetosonic Mach number, for the emissions related to a single FS.

\section{Discussion and conclusions}

We found that non-Io DAM emissions occur during both solar wind rarefaction and compression intervals (FS and RS related emissions), although on average the dynamic pressure was a factor of about 1.7 higher during the 2-days period surrounding the emissions than for the Ulysses distant encounter as a whole. There is also some connection to interplanetary shocks, with longer and more intense emissions occurring mainly in association with FS.

The observed brightening of the auroral radio emissions at a time of solar wind pressure increase is the opposite of the initial prediction based on corotation enforcement current system. But auroral brightening could follow a strong solar wind compression by the flywheel effect in the ionosphere (e.g., Cowley \& Bunce 2001; Clarke et al. 2009). Simulation results have shown that a transient brightening of the aurora can be expected, at slightly different latitudes, depending on the region of enhanced current, by the same principle of differential rotation rate of the plasma and field (Cowley et al. 2007). This has possibly been observed in the UV aurora (Nichols et al. 2007, 2009).

On the other hand, by analogy with studies of the Earth's magnetosphere, shock compression of the Jupiter's magnetosphere is likely to cause a strong electric-field acceleration of electrons along the auroral field lines. When a high-ram pressure interplanetary shock wave reaches the planet, the magnetosphere is suddenly compressed. As part of the effects, the strength and distribution of currents and plasma are modified, and enhanced fluxes of energetic charged particles are expected to precipitate into the auroral oval, which is then activated (Gurnett et al. 2002; Prangé et al. 2004; Clarke et al. 2009). One possible mechanism is a dayside aurora stimulated by shock compressions. Shocks can transfer energy from the interplanetary medium to the boundary layers and plasma, causing strong particle precipitation into the ionosphere and dayside auroral. This has been observed at Earth and is thought to occur also at Jupiter (Tsurutani et al. 2001).

Concerting the "efficiency" of solar wind structures to cause or be followed by non-Io DAM emissions, it was found that $71 \%$ of RS, $60 \%$ of FS and $58 \%$ of HCS are followed within one day by at least one emission. As a comparison, Echer \& Gonzalez (2004) studied the geoeffectiveness, for the Earth's magnetosphere, in terms of the Dst index, of several interplanetary structures. They found that a larger number of shocks were followed by moderate or intense storms, i.e., $57 \%$, than HCS (26\%). These results may imply that fast pressure changes have a higher longer impact on the auroral and magnetospheric processes than slower pressure changes. The former are expected to be accompanied by steep pressure gradients, which in turn could be associated with stronger auroral field aligned currents and related dissipation processes (e.g., Haerendel 2008).

Acknowledgements. Thanks to the Ulysses magnetometer and plasma sensor teams for the solar wind data. E. Echer thanks the FAPESP agency for financial support for this research (project 2007/52533-1). W.D.G. thanks FAPESP agency for financial support (project 2008/06650-9).

\section{References}

Acuna, M., Behannon, K. W., \& Connerney, J. P. 1983, in Physics of the jovian Magnetosphere, ed. A. J. Dessler (Cambridge University Press)

Anagnostopoulos, G. C., Louri, I., Marhavilas, P., \& Sarris, E. T. 2009, Adv. Space Res., 43, 573

Bagenal, F., Dowlilng, T. E., \& Mckinnon, W. B. 2004, in Jupiter, the planet, satellites and magnetospheres, ed. F. Bagenal, T. Dowling, \& W. McKinnon (Cambridge University Press)

Balogh, A., Beek, T. J., Forsyth, R. J., et al. 1992, A\&A, 92, 221

Bame, S. J., McComas, D. J., Barraclough, B. L., et al. 1992, A\&A, 92, 237

Barrow, C. H. 1978, Planetary Space Science, 26, 1193

Barrow, C. H. 1979, J. Geophys. Res., 84, 5366

Barrow, C. H., Genova, F., \& Desch, M. D. 1986, A\&A, 165, 244

Bigg, E. K. 1964, Nature, 203, 1008

Boischot, A. 1980, Icarus, 43, 399

Boischot, A., Sastri, J. H., \& Zarka, P. 1987, A\&A, 175, 287

Bozyan, E. A., \& Douglas, J. N. 1976, J. Geophys. Res., 81, 3387

Burke, B. F., \& Franklin, K. L. 1955, J. Geophys. Res., 60, 213

Carr, T. D., Desch, M. D., \& Alexander, J. K. 1983, in Physics of the jovian Magnetosphere, ed. A. J. Dessler (Cambridge University Press)

Clarke, J. T., Grodent, D., Cowley, S. W. H., et al. 2004, in Jupiter, the planet, satellites and magnetospheres, ed. F. Bagenal, T. Dowling, \& W. McKinnon (Cambridge University Press)

Clarke, J. T., Nichols, J., Gérard, J.-C., et al. 2009, J. Geophys. Res., 114, A 05210

Cowley, S. W. H., \& Bunce, E. J. 2001, Planet. Space Sci., 49, 1067

Cowley, S. W. H., Nichols, J. D., \& Andrews, D. J. 2007, Ann. Geophys., 25, 1433

Crary, F. J., \& Bagenal, F. 1997, Geophys. Res. Lett., 24, 2135

de Koning, C. A., Steinberg, J. T., Gosling, J. T., et al. 2005, J. Geophys. Res., 110, A01102 
Echer, E., \& Gonzalez, W. D. 2004, Geophys. Res. Lett., 31, L09808

Echer, E., et al. 2003, Braz. J. Phys., 33, 115

Echer, E., Tsurutani, B. T., \& Guarnieri, F. L. 2010, Adv. Spa. Res., 45, 798

Genova, F., Zarka, P., \& Barrow, C. H. 1987, A\&A, 182, 159

Genova, F., Zarka, P., \& Lecacheux, A. 1989, in Time-variable Phenomena in the Jovian System, ed. M. J. S. Belton, R. A. West, \& J. Rahe, NASA SP-494, 156

Gerard, J.-C., Saglam, A., Grodent, D., \& Clarke, J. T. 2006, J. Geo- phys. Res., 111(A10), A04202

Goldreich, P., \& Lynden-Bell, D. 1969, ApJ, 156, 59

Goldstein, M. L., \& Goertz, C. K. 1983, in Physics of the jovian Magnetosphere, ed. A. J. Dessler (Cambridge University Press)

Gurnett, D. A., Kurth, W. S., Hospodarsky, G. B., et al. 2002, Nature, 415, 985

Haerendel, G. 2008, J. Geophys. Res., 113, A07205

Ladreiter, H. P., Zarka, P., \& Lecacheux, A. 1994, Planet. Space Sci., 42, 919

Leblanc, Y., Gerbault, A., Denis, L., \& Lecacheux, A. 1993, A\&AS, 98, 529

MacDowall, R. J., Desch, M. D., Kaiser, M. L., et al. 2006, in Planetary Radio Astronomy VI, Proc. of the Sixth International Workshop, ed. H. O. Rucker, W. S. Kurth, \& G. Mann (Vienna: Austrian Academy of Sciences Press)
Morioka, A., Tsuchiya, F., Miyoshi, Y., et al. 2002, Earth Planets Space, 54, 1277

Nichols, J. D., Bunce, E. J., Clarke, J. T., et al. 2007, J. Geophys. Res., 112(A11), A02203

Nichols, J. D., Clarke, J. T., Gerard, J.-C., Grodent, D., \& Hansen, K. C. 2009, J. Geophys. Res., 114, A06210

Prangé, R., Zarka, P., Ballester, G. E., et al. 1993, J. Geophys. Res. -Planets, 98, 18779

Prangé, R., Pallier, L., Hansen, K. C., et al. 2004, Nature, 432, 78

Queinnec, J., \& Zarka, P. 1998, J. Geophys. Res., 103, 26649

Smith, E. J., \& Wolfe, J. H. 1976, Geophys. Res. Lett., 3, 137

Southwood, D. J., \& Kivelson, M. G. 2001, J. Geophys. Res., 106, 6123

Terasawa, T., Maezawa, K., \& Machida, S. 1978, Nature, 273, 131

Tsurutani, B. T. 2001, Surv. Geophys., 22, 101

Zarka, P. 1998, J. Geophys. Res., 103, 20159

Zarka, P., \& Genova, F. 1989, in Time-variable Phenomena in the Jovian System, ed. M. J. S. Belton, R. A. West, \& J. Rahe, NASA SP-494, 156

Zarka, P., Cecconi, B., \& Kurth, W. S. 2004, J. Geophys. Res., 109, A09S15 